\title{
Molecular and Serological Detection of Crimean-Congo Hemorrhagic Fever Virus in Sulaimani Province, Iraq
}

\author{
Tariq A. G. Aziz1, Dlovan J. Ali², Dilshad O. Jaff ${ }^{3}$ \\ ${ }^{1}$ Department of Microbiology, College of Medicine, Sulaimani University, Sulaymaniyah, Iraq \\ ${ }^{2}$ Department of Microbiology, College of Veterinary Medicine, Sulaimani University, Sulaymaniyah, Iraq \\ ${ }^{3}$ Gillings School of Global Public Health, University of North Carolina, Chapel Hill, NC, USA \\ Email: dr.taga@yahoo.com
}

Received 19 February 2016; accepted 16 April 2016; published 19 April 2016

Copyright (C) 2016 by authors and Scientific Research Publishing Inc.

This work is licensed under the Creative Commons Attribution International License (CC BY). http://creativecommons.org/licenses/by/4.0/

(c) (i) Open Access

\begin{abstract}
Background: Crimean-Congo hemorrhagic fever virus (CCHFV) is a member of the Nairovirus genus in the Bunyaviridae family and is transmitted by ticks of the Hyalomma genus. It causes severe disease in humans with mortality rates between $3 \%$ and $30 \%$. The first case in Iraq was reported in 1979; immediately thereafter, 10 cases were reported, resulting in seven deaths. Several cases were also reported in 1980 in Halabja city in Iraq's Sulaimani province. Methods: Blood samples were collected from two study populations, human and cattle, including butchers working at a slaughterhouse. Ticks were also collected for virus detection from three villages in the Sharazoor district of Sulaimani province. Results: Results of the Reverse Transcription-Polymerase Chain Reaction (RT-PCR) were negative for all human and cattle sera. The tissue prepared from ticks also was negative for CCHFV. An Enzyme-Linked Immunosorbent Assay (ELISA) technique showed that $30(46.87 \%)$ of 64 human sera were positive for the anti-CCHFV IgG antibody. Conclusion: Results show that symptomatic Crimean-Congo hemorrhagic fever (CCHF) is an uncommon disease in the Sulaimani province of Iraq and no clinical cases is reported, due to the eradication of ticks implemented by veterinary authorities. However, other preventive approaches and strategies should be implemented and monitored regularly by local authorities.
\end{abstract}

\section{Keywords}

\author{
CCHFV, CCHF, RT-PCR, ELISA
}




\section{Introduction}

Viral hemorrhagic fever (VHF) is a clinical illness that is becoming a serious threat to animals and humans [1]. One of many VHFs is Crimean-Congo hemorrhagic fever (CCHF), an acute, highly contagious, tick-borne viral zoonotic disease that is listed as a notifiable disease [2]. The disease is widely distributed globally with hemorrhagic manifestations and considerable mortality in humans, but no specific clinical symptoms in animals [3] [4]. The disease is caused by Crimean-Congo hemorrhagic fever virus (CCHFV), which is a member of the Nairovirus genus within the Bunyaviridae family. This family comprises more than 300 species grouped into five distinct genera. Members of the Bunyaviridae family are enveloped, single-stranded RNA genomes of negative polarity [5]. CCHFV has the most extensive geographic distribution of all tick-borne viruses and is widespread in Eurasia (including the Middle East) and Africa [6]. In Iraq, several cases were reported in 1979 with a high mortality rate, and all such individuals were in direct contact with domestic animals [7]-[9]. Infection with CCHFV in humans occurs through tick bites or direct contact with blood or tissues of infected livestock and patients. Workers in the livestock and agricultural industries and slaughterhouses, medical laboratory staff, and health care workers are at high risk [3]. The virus was first isolated from ticks of the Hyalomma genus during the 1960s. It has been detected in at least 31 species of ticks in the Ixodidae and Argasidae families [10]. A common pathogenic feature of CCHFV is its ability to disable host immune response [11]. The main pathological mechanism is believed to be vascular endothelial damage and the main target is thought to be the reticuloendothelial system [12].

In addition to its effects on animals, CCHFV is a main pathogen for humans. The most commonly observed symptoms and clinical signs in humans are fever, severe headache, dizziness, photophobia, myalgia, arthralgia, bleeding, ecchymosis, hematemesis, melena, epistaxis, haematuria, heamoptysis, nausea, and vomiting [13]. Early diagnosis of the virus is crucial to seeking treatment and to preventing and controlling further spread of the disease. Several methods are used for the diagnosis of CCHF such as Reverse Transcriptase-Polymerase Chain Reaction (RT-PCR) [14], Enzyme-Linked Immunosorbent Assay (ELISA) [15], and viral isolation [16]. The control and prevention of this viral disease requires controlling ticks [17], enhancing surveillance using standardized case definition, increasing laboratory capacity in endemic areas and areas at risk for CCHF expansion, awareness of prophylactic measures in the general population and in healthcare workers, and modifying their risk for infection [18] [19]. Vaccines for CCHFV are not available in most countries for either humans or animals [20].

The aim of this study was to detect CCHFV in humans, cattle, and ticks using molecular and serological methods. The research was conducted in Sulaimani province of the Kurdistan region, Iraq.

\section{Materials and Methods}

\subsection{Study Area and Sample Collection}

Sera collection was conducted between May 2014 and February 2015. The study was confined to three villages (Kaochktash, Kanipanka, and Darashesh) in the Sharazoor district of Sulaimani province (Figure 1), where cases of CCHF were reported in 1980.

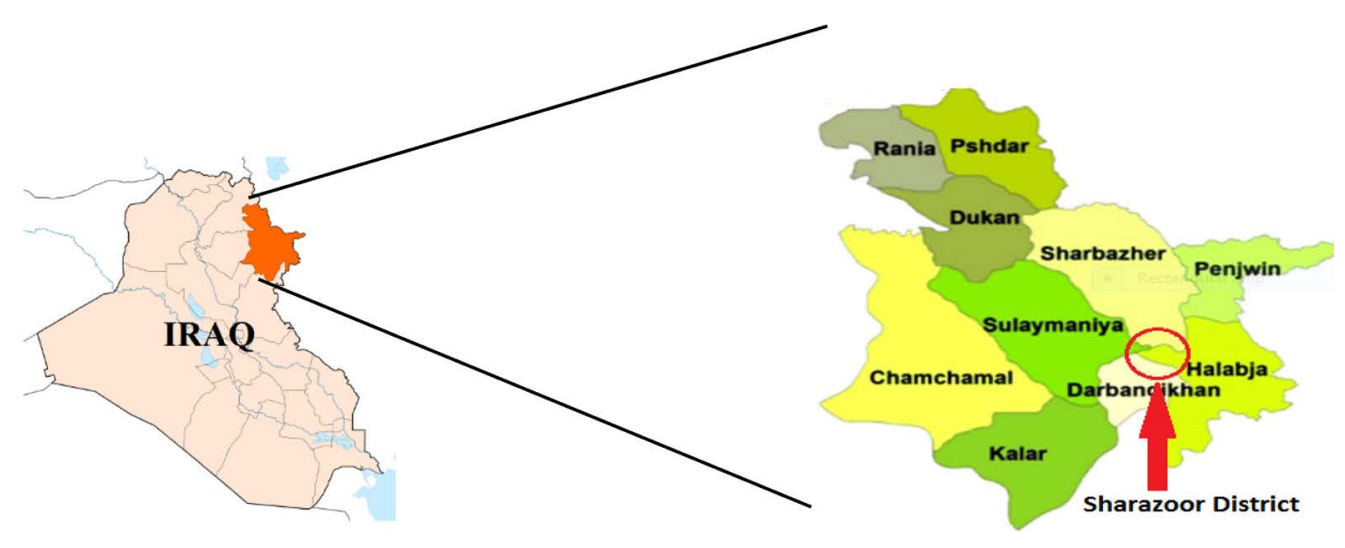

Figure 1. Study area: Sharazoor district of Sulaimani province. 
Random blood samples were collected from human and cattle, representing about $1 \%$ of the targeted population of human and cattle. Ninety-four human blood samples were collected and divided into two parts, the first for virus detection by RT-PCR and the second for detection of the anti-CCHFV antibody by ELISA. 110 blood samples were collected from cattle for virus detection by RT-PCR. A total of 55 ticks were also collected from cattle by forceps and placed into test tubes, which were then transported to the research laboratory for virus detection by RT-PCR. Sixteen serum samples were collected from an occupational risk group (butchers at slaughterhouses who were in contact with animals). As controls, 30 serum samples were collected from persons living in Sulaimani city center area who had never been in contact with animals.

\subsection{Nested RT-PCR for CCHFV Detection}

The extraction of viral RNA from human blood samples, cattle blood samples, and ticks was performed according to manufacturer procedures (the Geneaid Company, South Korea). Oligonucleotides were ordered from the Oligo (MACROGEN) Company, South Korea (Table 1).

\subsection{Detection of Anti-CCHFV Antibody}

The ELISA kit for detection of human anti-CCHFV IgG antibody was performed according to the manufacturer's procedures (Alpha Diagnostic International, Texas, USA). The results were determined using the following calculations: 1) The positive index was calculated as the net OD mean + 2SDs of the control samples. 2) The sample net OD mean was divided by the positive index. 3) Values above 1.0 were considered positive for the antibody and values below 1.0 considered negative.

\section{Statistical Analysis}

The Statistical Package for Social Science (SPSS) version 21 was used for analysis. Two approaches were used: descriptive and analytical. The descriptive approach includes the calculation of frequencies, percentages and means values. In the analytical approach, Chi-square test (X2) was used to test the association between categorical variables. The $\mathrm{P}$ value of equal or less than 0.05 was considered statistically significant.

\section{Results}

\subsection{Detection of CCHFV from Human and Animal Samples Using RT-PCR}

RT-PCR was used for the detection of CCHFV-RNA from blood samples collected from humans and cattle in the three villages; the results were negative for all 94 human and 110 cattle samples. The same technique was used to detect viral RNA from tissues prepared from 55 pooled ticks collected from cattle in the same area, and all were negative (Table 2). To confirm the accuracy of the results of the RT-PCR, two housekeeping genes (bovine and tick) were used and the result was negative.

\subsection{Seroprevalence of IgG Anti-CCHFV Antibody in Human Sera}

ELISA was used on negative human serum samples by RT-PCR to determine the immunological status of the

Table 1. Oligonucleatides (primers) for RT-PCR for the detection of Genes of CCHFV. F: Forward; R: Reverse.

\begin{tabular}{cclcc}
\hline \multicolumn{1}{c}{ Gene } & Polarity of Primer & \multicolumn{1}{c}{ Primer Sequence } & Amplicon Size bp & Primer Design \\
\hline \multirow{2}{*}{ CCHF Segment S gene } & $\mathrm{F}$ & CTG CTC TGG TGG AGG CAA CAA & 452 & NCBI \\
& $\mathrm{R}$ & TGG GTT GAA GGC CAT GAT T & NCBI \\
CCHF Segment S gene & $\mathrm{F}$ & AGG TTT CCG TGT CAA TGC AAA & 207 & NCBI \\
Beta actin mRNA & $\mathrm{R}$ & TTG ACA AAC TCC CTG CAC CAG T & & NCBI \\
Glycerald ehyde-3- & $\mathrm{F}$ & ATG TGT GAC GAC GAG GTT GCC GC & 432 & \\
phosphate & $\mathrm{R}$ & GTA CAG CGA CAG CAC GGC CTG G & 452 & NCT \\
dehydrogenase mRNA & $\mathrm{F}$ & CCA CTC CCA ACG TGT CTG TT & & \\
\hline
\end{tabular}


population in this study: those who have daily contact with cattle or who are members of the risk group (butchers from a Sulaimani slaughterhouse), and a control group population of 30 young students (aged 20 - 30) from University of Sulaimani who were never in contact with cattle, according to the data collection sheet. The overall anti-CCHFV IgG antibody prevalence among the residents of the three villages was $46.87 \%$ (30/64). The sex prevalence for females was $36.7 \%(11 / 30)$ and $63.3 \%$ (19/30) for males; no significant statistical differences were found between males and females $(\mathrm{p}=0.515)$. All 30 serum samples from young students were seronegative for the CCHFV IgG antibody, while serum samples obtained from butchers revealed anti-CCHFV IgG prevalence of $18.75 \%$ (3/16). Seroprevalence of the anti-CCHFV IgG antibody was $47.36 \%$ (9/19), 66.66\% (10/15) and 57.14\% (8/14) in farmers who had daily contact with cattle in Kaochktash, Kanipanka, and Darashesh villages, respectively (Table 3). There was a significant difference between risk groups and the control group ( $\mathrm{p}=$ $0.001)$.

\subsection{The Relationship between Age Groups and the Prevalence of the Anti-CCHFV Antibody}

The highest antibody prevalence was found among those aged 30 - 40 (56.25\%) and the lowest prevalence was found among the 40 - 50 year and 50 - 60 year age groups (43.75\%), while those aged 20 - 30 were seronegative for the IgG antibody ( $\mathrm{p}=0.001)$ (Table 4$)$.

\section{Discussion}

The apparent increase in the global distribution of CCHFV coupled with its circulation in numerous vertebrate species and a significant increase in case fatality rates associated with nosocomial transmission of CCHFV highlight the importance of a rapid, robust, and reliable diagnostic procedure to confirm the presence of the virus. Assays that lead to early diagnosis of CCHF will result in better prevention, patient management, isolation, and infection control strategies [21]. Serological and epidemiological surveys usually determine the endemicity of

Table 2. The results of RT-PCR from human, cattle and tick samples.

\begin{tabular}{cccc}
\hline Samples & Number of Samples & Positive Cases Number (Percent) & Negative Cases Number (Percent) \\
\hline Human & 94 & $0(0.0)$ & $94(100.0)$ \\
Cattle & 110 & $0(0.0)$ & $110(100.0)$ \\
Tick & 55 & $0(0.0)$ & $55(100.0)$ \\
\hline
\end{tabular}

Table 3. Seroprevalence of IgG anti-CCHFV antibody from human sera.

\begin{tabular}{ccccc}
\hline Location & Number of Samples & Positive Cases Number (Percent) & Negative Cases Number (Percent) & p Value \\
\hline Kaochktash Village & 19 & $9(47.36)$ & $10(52.63)$ & $5(33.3)$ \\
Kanipanka Village & 15 & $10(66.66)$ & $6(42.85)$ & $0.001^{*}$ \\
Darashesh Village & 14 & $8(57.14)$ & $13(81.25)$ \\
Slaughterhouse & 16 & $3(18.75)$ & $30(100.0)$ \\
City Center & 30 & $0(0.0)$ & $64(68.0)$ \\
Total & 94 & $30(31.91)$ & \\
\hline
\end{tabular}

*Significant at $\mathrm{p}<0.05$.

Table 4. Age distribution in relation to serum anti-CCHFV antibodies.

\begin{tabular}{cccc}
\hline Age Group & Number of Samples & Positive Cases Number (Percent) & Negative Cases Number (Percent) \\
\hline $20-30$ & 30 & $0(0.0)$ & $30(100.0)$ \\
$30-40$ & 16 & $9(56.25)$ & $7(43.75)$ \\
$40-50$ & 32 & $14(43.75)$ & $18(56.25)$ \\
$50-60$ & 16 & $7(43.75)$ & $9(56.25)$ \\
Total & 94 & $30(31.91)$ & $64(68)$ \\
\hline
\end{tabular}

\footnotetext{
${ }^{*}$ Significant at $\mathrm{p}<0.05$.
} 
viral diseases, including CCHF, in a particular region. In endemic areas, assessment of human and livestock antibodies for CCHFV appear to be one of the best indicators of CCHF risk. Standardized diagnostic methods, increasing public awareness through health education programs, and enhancing the capacity of health and veterinary professionals should be identified as priorities in endemic countries like Iraq, as CCHF is endemic in other areas of the country [9]. Ensuring good administrative arrangements and maximum cooperation between different authorities, particularly between health and veterinary authorities, are also essential.

The virus is transmitted by various tick species, but mainly those of the Hyalomma genus. Domestic ruminants are infected through tick bites, and can infect more ticks to perpetuate the virus [22] [23]. In rural areas in the Kurdistan region of Iraq, agriculture, animal husbandry, free grazing, uncontrolled trade, movement of sheep and goats (especially in border areas), poor farm hygiene, and lack of education and knowledge are among the factors contributing to the wide spread and transmission of the infection and challenging its complete eradication.

As shown in Table 2, the RT-PCR results for human, cattle, and tick samples were negative for the CCHFV genome. To confirm the reliability of RT-PCR in our study, two housekeeping genes were used to analyze the relative expression of different genes as indicators of perfect nucleic acid extraction, quality of samples and quality of PCR. The results were positive for both housekeeping genes and were negative for all samples tested. This diminishes the possibility of false negative results. The selection of specific viral primers made the technique more specific. The primers were chosen from highly conserved fragments of the extreme terminal regions of the S-segment, which is more likely to detect all strains of the virus. It is well documented that the CCHFV and RNA S-segment, which codes for viral nucleoprotein (NP) and non-structural proteins, have fewer variable nucleotide sequences among cognate genes of CCHFV strains [24].

One of the most striking concerns about the geographical distribution of CCHFV is the lack of clinical cases in the Western Mediterranean, west of the main distribution area of the pathogen. This is of special interest because the main tick vector Hyalomma marginatum is abundant in places like Spain and Southern Italy, as well as in Mediterranean and African countries where neither the disease nor the virus have been reported [25]. Thirty-six years ago, CCHF was endemic in the study area where clinical cases were reported, but since 1997 there have been no reports of human infection cases of the disease in Sulaimani province according to data from the directorate of health and protection in Sulaimani province. It would appear that CCHF is not currently a serious health problem in this province. In this study, molecular analysis showed that ticks collected from the three villages were CCHFV-negative, which was reflected in the negative results in human and cattle samples. The absence of infection may be in part due to better eradication of ticks, which are the main vector and reservoir for the virus. The regular implementation of dipping and the use of acaricide on livestock have greatly reduced the vector population and the reservoir for the CCHFV, and are interrupting the life cycle of ticks and the virus.

In this paper, the prevalence of the anti-CCHFV IgG antibody, which is indicative of previous exposure to the virus, was $46.87 \%$. The failure of the public health system to detect acute cases may be attributable to the virus' non-specific clinical presentation, which makes it difficult to differentiate CCHF from other endemic cases of febrile illness. Strains of CCHFV like AP92 cause a much milder form of human disease and may represent a predominantly moderate severity of infection [26] [27].

As shown in Table 4, those aged 30 - 40 had the highest (56.52\%) seroprevalence rate. This finding is comparable to findings reported in Iran and Kenya [28] [29] and confirms the important risk factors for CCHFV exposure, which include being in high risk occupations (farming and butchering) where individuals have contact with livestock, and being over the age of 30. In this study, comparing the seroprevalence of risk groups that had daily contact with livestock (46.87\%) to the control group (0\%), who are young students living in urban areas, showed that there are highly significant differences between them $(\mathrm{p}<0.05)$. This finding corresponds with findings observed in Kenya and South Africa [29] [30] and is consistent with studies carried out in Africa, Europe and the Middle East, which showed that people working with livestock, such as farmers and butchers, have the highest prevalence of anti-CCHFV IgG antibody, indicating their high risk of infection [28]. Despite reports of the disease from neighboring countries such as Iran (mostly from areas closer to the Pakistani border), Russia, and Bulgaria, the number of reported cases in these countries is much higher than in Iraq [31]. It is unclear when and how seropositive humans in this study were infected without any appearance of disease or clinical symptoms, but they may have been in contact with infected ticks or cattle early in life (before 1997), become subclinically infected, and seropositive thereafter. 


\section{Conclusion}

According to the data and findings presented in this study, Sulaimani province has become an area in which CCHF is not currently a serious health problem. However, more efforts should be made by health and veterinary authorities in this province to increase public awareness of the disease and to prepare to properly assess and treat any future cases. These recommendations can be achieved with multidisciplinary, integrated, and collaborative approaches.

\section{Acknowledgements}

Thanks to the Department of Medical Microbiology, School of Medicine, Sulaimani University, Iraq, for their cooperation and support.

\section{References}

[1] Mardani, M. and Jahromi, M.K. (2007) Crimean-Congo Hemorrhagic Fever. Archives of Iranian Medicine, 10, 204214.

[2] World Organization for Animal Health (WHO) (2016) OIE-Listed Disease, Infections and Infestations in Force in 2016. http://www.oie.int/animal-health-in-the-world/oie-listed-diseases-2016/

[3] Faghihi, F., Chinikar, S., Telmadarraiy, Z., et al. (2015) Crimean-Congo Hemorrhagic Fever: A Seroepidemiological and Molecular Survey in North of Iran. Journal of Entomology and Zoology Studies, 3, 156-159

[4] Chinikar, S., Moghadam, A.H., Parizadeh, S.M.J., et al. (2012) Seroepidemiology of Crimean Congo Hemorrhagic Fever in Slaughterhouse Workers in North Eastern Iran. Iranian Journal of Public Health, 41, 72-77.

[5] Clerex-van Haaster, C.M., Clerex, J.P., Ushijima, H., Akashi, H., Fuller, F. and Bishop, D.H. (1982) The 3 Terminal RNA Sequences of Bunyaviruses and Nairoviruses (Bunyaviridae): Evidence of End Sequence Generic Differences within the Virus Family. Journal of General Virology, 61, 33-34. http://dx.doi.org/10.1099/0022-1317-61-2-289

[6] Maltezou, H.C. and Papa, A. (2011) Crimean-Congo Hemorrhagic Fever: Epidemiological Trends and Controversies in Treatment. BMC Medicine, 9, 131. http://dx.doi.org/10.1186/1741-7015-9-131

[7] Al-Tikriti, S.K., Al-Ani, F., Jurji, F.J., Tantawi, H., Al-Mosleh, M., Al-Janabi, N., et al. (1981) Congo-Crimean Hemorrhagic Fever in Iraq. Bulletin of the World Health Organization, 59, 85-90.

[8] Tantawi, H.H., Al-Mosleh, M.I., Al-Janabi, N.Y., Al-Bana, A.S., Mahmud, M.I., Jurji, F., Yonan, M.S., Al-Ani, F. and Al-Tikriti, S.K. (1980) Crimean-Congo Hemorrhagic Fever Virus in Iraq: Isolation, Identification and Electron Microscopy. Acta Virologica (Praha), 24, 464-467.

[9] Emad, S.A., Nabeel, A.M. and Sumad, M.W. (2012) Crimean-Congo Hemorrhagic Fever in Iraq during 2010. Proceeding of the Eleventh Veterinary Scientific Conference, Iraq, 99-103.

[10] Kayedi, M.H., Chinikar, S., Mostafavi, E., et al. (2015) Crimean-Congo Hemorrhagic Fever Virus Clade IV (Asia 1) in Ticks of Western Iran. Journal of Medical Entomology, 52, 1144-1149. http://dx.doi.org/10.1093/jme/tjv081

[11] Bereczky, S., Lindegren, G., Karlberg, H., Akerstrom, S., Klingstrom, J. and Mirazimi, A. (2010) Crimean-Congo Hemorrhagic Fever virus Infection Is Lethal for Adult Type I Interferon Receptor-Knockout Mice. Journal of General Virology, 91, 1473-1477. http://dx.doi.org/10.1099/vir.0.019034-0

[12] Marty, A.M., Jahrling, P.B. and Geistbert, T.W. (2006) Viral Hemorrhagic Fevers. Clinics in Laboratory Medicine, 26, 345-386, viii. http://dx.doi.org/10.1016/j.cll.2006.05.001

[13] Baraiteanu, S., Danes, D., Dumitrescu, G.V., Ionescu, L.E. and Vladimirescu, A.F. (2015) Molecular Biology Techniques Used in Active Surveillance of Crimean-Congo Haemorrhagic Fever in Ruminants: A Critical Review. Scientific Bulletin Series F. Biotechnologies, XIX, 3-96.

[14] Duh, D., Saksida, A., Petrovec, M., Dedushaj, I. and Avsic-Zupanc, T. (2006) Novel one Step Real-Time RT-PCR Assay for Rapid and Specific Diagnosis of Crimean-Congo Hemorrhagic Fever Encountered in the Balkans. Journal of Virological Methods, 133, 175-179. http://dx.doi.org/10.1016/j.jviromet.2005.11.006

[15] Yapar, M., Aydogan, H., Pahsa, A., Besirbellioglu, B.A., Bodur, H. and Basustaoglu, A.C. (2005) Rapid and Quantitative Detection of Crimean-Congo Hemorrhagic Fever Virus by one Step Real-Time RT-PCR. Japanese Journal of Infectious Diseases, 58, 358-362.

[16] Shephered, A.J., Swanepoel, R., Leman, P.A. and Shephered, S.P. (1986) Comparison of Methods for Isolation and Titration of Crimean-Congo Hemorrhagic Fever Virus. Journal of Clinical Microbiology, 24, 654-656.

[17] Keshtkar-Jahromi, M., Kuhn, J.H., Christova, I., Bradfute, S.B., Jahrling, P.B. and Bavari, S. (2011) Crimean-Congo Hemorrhagic Fever: Current and Future Prospects of Vaccines and Therapies. Antiviral Research, 90, 85-92. 
http://dx.doi.org/10.1016/j.antiviral.2011.02.010

[18] Maltezou, H.C., Andonova, L., Andraghetti, R., Bouloy, M., Ergonul, O., Jongejan, F., Kalvatchev, N., Nichol, S., Niedrig, M., Platonov, A., Thomson, G., Leitmeyer, K. and Zeller, H. (2010) Crimean-Congo Hemorrhagic Fever in Europe: Current Situation Calls for Preparedness. Eurosurveillance, 15, 1-4.

[19] Bajpai, S. and Nadkar, M.Y. (2011) Crimean-Congo Hemorrhagic Fever: Requires Vigilance and Not Panic. Journal of the Association of Physicians of India, 59, 164-167.

[20] Papa, A., Papadimitriou, E. and Christova, I. (2011) The Bulgarian Vaccine Crimean-Congo Hemorrhagic Fever Virus Strain. Scandinavian Journal of Infectious Diseases, 43, 225-229. http://dx.doi.org/10.3109/00365548.2010.540036

[21] Atkinson, B., Chamberlain, J., Logue, C.H., Cook, N., Bruce, C., Dowall, S.D. and Hewson, R. (2012) Development of a Real-Time RT-PCR Assay for the Detection of Crimean-Congo Hemorrhagic Fever Virus. Vector-Borne and Zoonotic Diseases, 12,786-793. http://dx.doi.org/10.1089/vbz.2011.0770

[22] Hoogstraal, H. (1979) The Epidemiology of Tick-Borne Crimean-Congo Hemorrhagic Fever Virus in Asia, Europe, and Africa. Journal of Medical Entomology, 15, 307-417. http://dx.doi.org/10.1093/jmedent/15.4.307

[23] Whitehouse, C.A. (2004) Crimean-Congo Hemorrhagic Fever. Antiviral Research, 64, 145-160. http://dx.doi.org/10.1016/S0166-3542(04)00163-9

[24] Deyde, V.M., Khristova, M.L., Rollin, P.E., Ksiazek, T.G. and Nichol, S.T. (2006) Crimean-Congo Hemorrhagic Fever Virus Genomics and Global Diversity. Journal of Virology, 80, 8834-8842. http://dx.doi.org/10.1128/JVI.00752-06

[25] Estrada-Pen, A., Lisa, J., Jolyon, M., Zati, V. and Farida, T. (2012) Unraveling the Ecological Complexities of Tick-Associated Crimean-Congo Hemorrhagic Fever Virus Transmission: A Gap Analysis for the Western Palearctic. Vectoe-Borne and Zoonotic Diseases, 12, 743-752. http://dx.doi.org/10.1089/vbz.2011.0767

[26] Antoniadis, A. and Casals, J. (1982) Serological Evidence of Human Infection with Congo-Crimean Hemorrhagic Fever Virus in Greece. The American Journal of Tropical Medicine and Hygiene, 31, 1066-1067.

[27] Ozkaya, E., Dincer, E., Carhan, A., Uyar, Y., Ertek, M. and Whitehouse, C.A. (2010) Molecular Epidemiology of Crimean-Congo Hemorrhagic Fever Virus in Turkey: Occurrence of Local Topotype. Virus Research, 149, 64-70. http://dx.doi.org/10.1016/j.virusres.2009.12.014

[28] Izadi, S., Holakouie-Naieni, K. and Madjdzadeh, S.R. (2004) Crimean-Congo Hemorrhagic Fever in Sistan and Balouchestan Province of Iran: A Case Control Study on Epidemiological Characteristics. International Journal of Infectious Diseases, 8, 299-306. http://dx.doi.org/10.1016/j.ijid.2003.10.008

[29] Olivia, W.L., Zephania, I., Caroline, T., Edith, C., Benedict, O., Lillian, M., Marietjie, V., Anne, F. and Rosemary, S. (2012) Seroprevalence of Crimean-Congo hemorrhagic Fever Virus in Ijara District, Kenya. Vector-Borne and Zoonotic Diseases, 12, 727-732. http://dx.doi.org/10.1089/vbz.2011.0914

[30] Fisher-Hoch, S.P., McCormick, J.B. and Swenpopel, R. (1992) Risk of Human Infection with Crimean-Congo Hemorrhagic Fever Virus in South Africa Rural Community. The American Journal of Tropical Medicine and Hygiene, 47, 337-345.

[31] Alavi-Naini, R., Moghtaderi, A. and Koohpayeh, H.R. (2006) Crimean-Congo Hemorrhagic Fever in Southeast of Iran. Journal of Infection, 52, 378-382. http://dx.doi.org/10.1016/j.jinf.2005.07.015 